\title{
AAV-Mediated angiotensin 1-7 overexpression inhibits tumor growth of lung cancer in vitro and in vivo
}

\author{
Xinglu Chen ${ }^{1, *}$, Sansan Chen ${ }^{2, *}$, Nana Pei $^{3, *}$, Yingying Mao ${ }^{1}$, Shengyao Wang ${ }^{1}$, \\ Renhe Yan ${ }^{1}$, Na Bai ${ }^{4}$, Andrew Li ${ }^{5}$, Yanling Zhang ${ }^{1}$, Hongyan Du${ }^{1}$, Baihong Chen ${ }^{1}$, \\ Colin Sumners ${ }^{6}$, Jinlong $\mathbf{L i}^{1}$, Hongwei $\mathbf{L i}^{1}$ \\ ${ }^{1}$ School of Laboratory Medicine and Biotechnology, Southern Medical University, Guangzhou, Guangdong, China \\ ${ }^{2}$ Department of Urology, The First Affiliated Hospital of Clinical Medicine of Guangdong Pharmaceutical University, Guangzhou, \\ Guangdong, China \\ ${ }^{3}$ Department of Clinical Pathology, The First Affiliated Hospital of Jinan University, Guangzhou, Guangdong, China \\ ${ }^{4}$ Deparement of Nuclear Medicine, People's Hospital of Yuxi City, Yuxi, Yunnan, China \\ ${ }^{5}$ Department of Biomedical Engineering, The Johns University School of Medicine, Baltimore, USA \\ ${ }^{6}$ Departments of Physiology and Functional Genomics, University of Florida, Gainesville, Florida, USA \\ *These authors contributed equally to this work
}

Correspondence to: Hongwei Li, email: hongwei1@yahoo.com Jinglong Li, email: lijinlong75@126.com

Keywords: Ang-(1-7), lung cancer, adeno-associated viral vector, DNA synthesis, proliferation

Received: October 07, 2016

Accepted: November 11, 2016

Published: November 16, 2016

\section{ABSTRACT}

Ang-(1-7) inhibits lung cancer cell growth both in vitro and in vivo. However, the molecular mechanism of action is unclear and also the rapid degradation of Ang-(1-7) in vivo limits its clinical application. Here, we have demonstrated that Ang-(1-7) inhibits lung cancer cell growth by interrupting pre-replicative complex assembly and restrains epithelial-mesenchymal transition via Cdc6 inhibition. Furthermore, we constructed a mutant adeno-associated viral vector AAV8 (Y733F) that produced stable and high efficient Ang-(1-7) expression in a xenograft tumor model. The results show that AAV8-mediated Ang-(1-7) over-expression can remarkably suppress tumor growth in vivo by down-regulating Cdc6 and antiangiogenesis. Ang-(1-7) over-expression via the AAV8 method may be a promising strategy for lung cancer treatment.

\section{INTRODUCTION}

Non-small cell lung cancer (NSCLC) accounts for over $80 \%$ of all lung cancers, and remains the most common cause of cancer-related death $[1,2]$. Chemotherapy has been traditionally considered as one of the standard treatment options for advanced NSCLC, but the toxic side-effects and chemo-resistance hinder its clinical application [1]. Gene therapy has become a promising strategy for lung cancer therapies.

Ang-(1-7), an endogenous heptapeptide hormone of the renin-angiotensin system, mediates biological responses by activating Mas, a unique $\mathrm{G}$ protein-coupled receptor. Studies on Ang-(1-7) and Mas have mainly focused on hypertension and other cardiovascular diseases [3]. Interestingly, mounting evidence has shown that Ang-(1-7) exerts potent antitumor effects mainly through inhibitory effects on DNA synthesis, migration and invasion, and also antiangiogenic actions in a variety of tumors $[4,5]$. Phase I/II dose escalation studies have assessed the safety and activity of Ang-(1-7) following chemotherapy in patients with breast cancer [6, 7]. However, the mechanisms underlying impairment of tumor proliferation are still unclear. Besides, the efficacy of this peptide in vivo is severely hampered due to rapid degradation by peptidases [7].

Here, we confirmed the inhibitory effect of Ang(1-7) on proliferation and migration of NSCLC cells and demonstrated that the mechanism may be associated with suppressing pre-replicative complex (pre-RC) assembly and epithelial-mesenchymal transition (EMT). Moreover, we constructed a recombinant Adeno- 
associated virus (rAAV) vector to overexpress Ang-(1-7) continuously and stably in a murine xenotransplantation model of a human non-small cell lung cancer cell line (Spc-A1). The results indicate that overexpression of Ang-(1-7) inhibits tumor growth by decreasing Cdc6 and reduces angiogenesis by down-regulating VEGF.

\section{RESULTS}

\section{Ang-(1-7) inhibits lung cancer cell proliferation by interrupting pre-RC assembly}

To verify the inhibitory effect of Ang-(1-7) on lung cancer cells, A549 and Spc-A1 cells were infected with lentiviral vector LV-Ang-(1-7) or LV-eGFP at $200 \mathrm{vg} / \mathrm{cell}$ respectively. Transfected cells were plated into a 24 -well cluster dish and then were counted on day 5, 6, 7 and 8 to determine the cell growth. The data demonstrated that cell growth was inhibited in the LV-Ang-(1-7) transduced cells compared to the LV-eGFP or mock transduced cells (Figure 1).

A BrdU incorporation assay was used to determine whether Ang-(1-7) inhibited DNA replication of lung cancer cell lines. A549 and Spc-A1 lung cancer cells were treated with $500 \mathrm{nM}$ Ang-(1-7) for $24 \mathrm{~h}$. Next, $10 \mu \mathrm{M}$ BrdU was added and incubated with cells for $2 \mathrm{~h}$, followed by immunofluorescence detection (Figure 2A). As shown in the summary data in Figure 2B, the number of BrdU positive cells in the Ang-(1-7) group was significantly lower than in control groups, indicating that Ang-(1-7) reduced DNA synthesis. It has been demonstrated that Ang(1-7) exerts its biological effects by binding to the Mas receptor $[8,9]$. A779, a Mas receptor antagonist, attenuated the inhibitory effect of Ang-(1-7) on DNA synthesis.

Cdc6 plays a crucial role in the assembly of prereplication complexes (pre-RCs) by linking the origin recognition complex (Orc) with minichromosome maintenance $(\mathrm{Mcm})$ proteins to form pre-RCs at the sites of DNA replication. RT-PCR results from lung cancer Spc-A1 cells showed that Cdc6 mRNA level was significantly decreased by Ang-(1-7) (Figure 3A). Accordingly, Western blot analysis demonstrated that Ang-(1-7) decreased Cdc6 protein level in Spc-A1 cells (Figure 3B). In addition, $\mathrm{Mcm} 2$, a component of pre-RCs, was also down-regulated following Ang-(1-7) treatment (Figure 3B). Therefore, the data suggest that Ang-(1-7) may inhibit pre-RCs assembly, and point to a crucial role of $\mathrm{Cdc} 6$ and $\mathrm{Mcms}$ in this process. Down-regulation of Cdc6 mRNA and Cdc6 and Mcm2 proteins by Ang(1-7) were abolished by the Ang-(1-7) antagonist, A779 (Figure 3A, 3B).

To further investigate the role of Ang-(1-7) in preRCs assembly, we analyzed the expression of pre-RCs in the chromatin-binding fraction. As shown in Figure 3C, Mcm6, and Mcm7 loaded on chromatin were significantly decreased after Ang-(1-7) treatment, effects that were abolished by A779. These results suggest that Ang-(1-7) can disrupt pre-RCs assembly and subsequently inhibit DNA synthesis.

\section{Ang-(1-7) suppresses EMT in NSCLC}

It has been demonstrated that $\mathrm{Cdc} 6$ promotes epithelial to mesenchymal transition (EMT) [10]. Thus, we hypothesized that Ang-(1-7) may inhibit EMT in NSCLC due to its role in lowering Cdc6 expression and analyzed the protein levels of several epithelial and mesenchymal markers. Accordingly, the protein level of the epithelial marker E-cadherin was elevated by Ang-(1-7) treatment, while the levels of vimentin, a mesenchymal marker, were not altered (Figure 4A).

Tumor cells undergoing EMT tend to lose cell-cell adhesiveness [11]. Since Ang-(1-7) can restrain EMT in NSCLC cells, it may exert inhibitory effects on migration. Consistent with our speculation, the migration ability of Spc-A1 cells declined after Ang-(1-7) treatment, an effect that was blocked by the Mas antagonist, A-779 (Figure 4B).

\section{Ang-(1-7) inhibits p38 MAPK signaling pathway}

The p38 MAPK signaling pathway plays a vital role in cell proliferation and migration. The p38 phosphorylation was decreased by Ang-(1-7) treatment as compared with the saline group, an effect that was reversed by A779 (Figure 5).

\section{Ang-(1-7) inhibits lung tumor growth via anti- proliferative and anti-angiogenic effects}

The short elimination half time of Ang-(1-7) in vivo limits its clinical use [7]. Hence, we constructed an AAV vector, AAV-Ang-(1-7), to express the Ang-(1-7) consistently and stably. When xenograft tumor volumes were $\sim 50 \mathrm{~mm}^{3}$, mice were treated with AAV-Ang-(1-7), AAV-eGFP $\left(1 \times 10^{11} \mathrm{vg} /\right.$ mice $\left./ 100 \mu \mathrm{l}\right)$ or PBS by a single intravenous injection into the tail. Tumor volumes were measured every 3-days for a period of 34 days. The tumor volume in the AAV-Ang-(1-7)-treated group was significantly smaller than in AAV-eGFP and PBS groups from 19 days after injection, while there were no differences between the AAV-eGFP and PBS groups (Figure 6A). At the end of the study, the tumor weight and size in AAV-Ang-(1-7) group was markedly reduced compared with the control groups (Figure 6B, 6D).

To verify that AAV-Ang-(1-7) produced steady Ang-(1-7) expression, the Ang-(1-7) secreted into the serum was quantified by ELISA. The results showed that Ang-(1-7) was significantly increased in AAV-Ang(1-7)-treated mice compared with the AAV-eGFP or PBS groups (Figure 6C). Because of AAV mediated Ang-(1-7) expression targeting liver, fusion protein containing mouse $\mathrm{IgG} \mathrm{Fc}$ region could be detected via 
immunostaining using horseradish peroxidase-conjugated anti-mouse IgG (Figure 6E).

Tumor paraffin sections were stained with $\mathrm{Ki67}$ antibodies as shown in Figure 7A. Ki67 is a nuclear protein presented in the active phases of the cell cycle (G1, S, G2, and M), but is not expressed in quiescent cells. Tumor sections from Ang-(1-7)-treated animals stained with anti-Ki67 antibodies showed a significantly less percentage of stained cells compared with the two control groups, suggesting that Ang-(1-7) reduces Spc-A1 xenograft tumor cell proliferation.

High levels of Cdc6 have been reported in around $50 \%$ of non-small cell lung carcinomas [12], brain cancer
[13] and a subset of mantle cell lymphomas [14], which suggests that Cdc6 has oncogenic properties. Tumor sections stained with anti-Cdc6 showed significantly decreased Cdc6 immunostaining following treatment with Ang-(1-7) (Figure 7B).

\section{Ang-(1-7) reduces vessel density in Spc-A1 xenograft tumors}

In order to identify whether AAV-Ang-(1-7) inhibits angiogenesis within lung tumor xenografts, blood vessels were detected by immunohistochemical staining using VEGF and CD34 antibodies. Tumor sections stained
A

A549

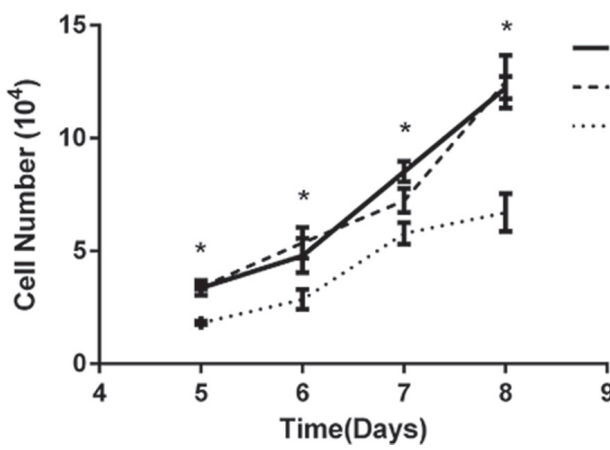

Spc-A1

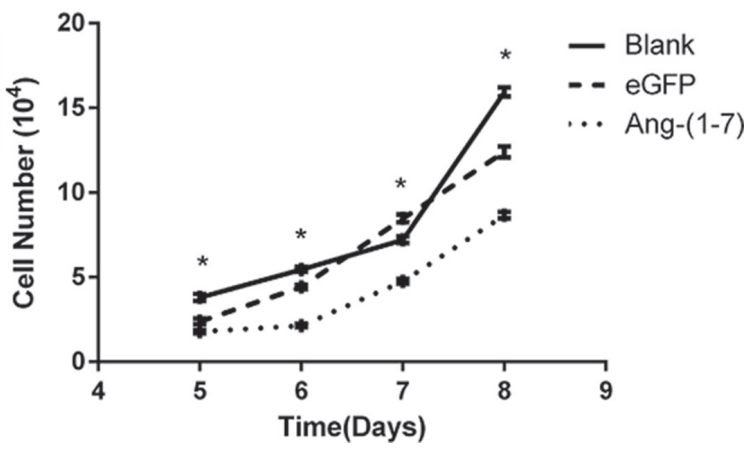

Figure 1: Ang-(1-7) inhibited growth of lung cancer cells. A549 (A) or Spc-A1 (B) cells were infected with LV- Ang-(1-7) or LV-eGFP. Effect of Ang-(1-7) on cell proliferation was counted from day 5 after cells were plated. $* P<0.01$ versus the control groups (LV-eGFP- and mock-transduced cells).

A

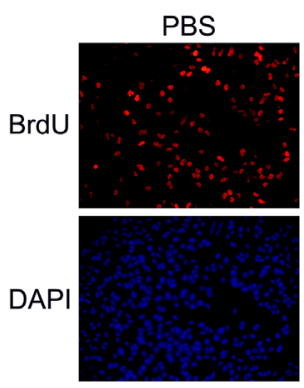

B

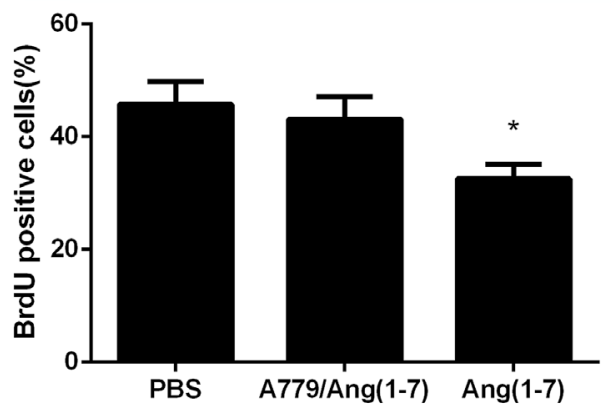

A549
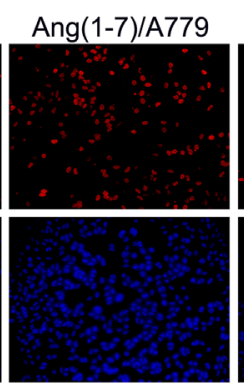

A549
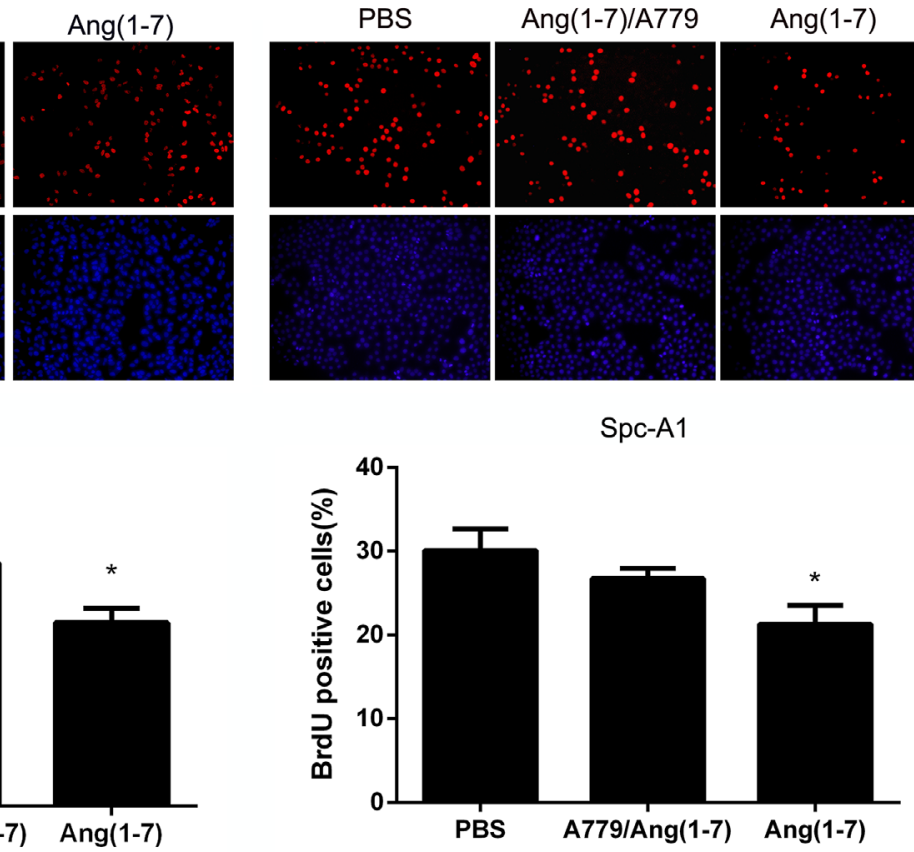

Figure 2: Ang-(1-7) inhibited DNA synthesis in lung cancer cells. (A) A549 and Spc-A1 cells were treated with PBS or $500 \mathrm{nmol} / \mathrm{L}$ Ang-(1-7) with or without $1 \mu \mathrm{mol} / \mathrm{L}$ A779 for $24 \mathrm{~h}$, then incubated with BrdU for $2 \mathrm{~h}$. (B) The BrdU-positive cells were identified by immunofluorescence staining and observed under a fluorescence microscope. $* P<0.05$. 

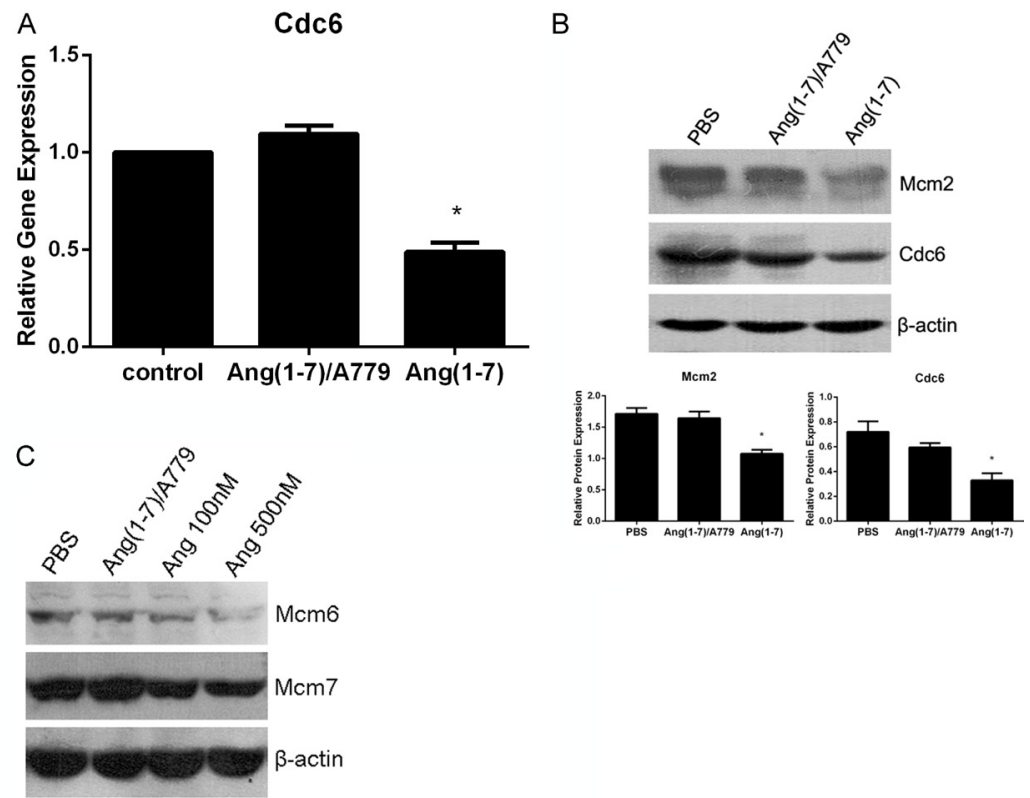

Figure 3: Ang-(1-7) downregulates Cdc6 mRNA and protein levels in Spc-A1 cells. Spc-A1 cells were treated with PBS or $500 \mathrm{nM}$ Ang-(1-7), with or without $1 \mu \mathrm{M}$ A779 for $24 \mathrm{~h}$. Cells were collected and subjected to real-time RT-PCR and Western blot analyses (data are representative of three different experiments). (A) Expression level of Cdc6 in Spc-A1 cells after treatment with Ang-(1-7) compared to control groups. ${ }^{*} P<0.01$. (B) Expression of Cdc6 and Mcm2 along with $\beta$-actin protein in Spc-A1 cells. Western blots are representative of three different experiments. ${ }^{*} P<0.01$. (C) Expression of Mcm6 and Mcm7 protein in Spc-A1 cells treated with PBS, $1 \mu \mathrm{M}$ A779 with $500 \mathrm{nM}$ Ang-(1-7), $100 \mathrm{nM}$ or $500 \mathrm{nM}$ Ang-(1-7).

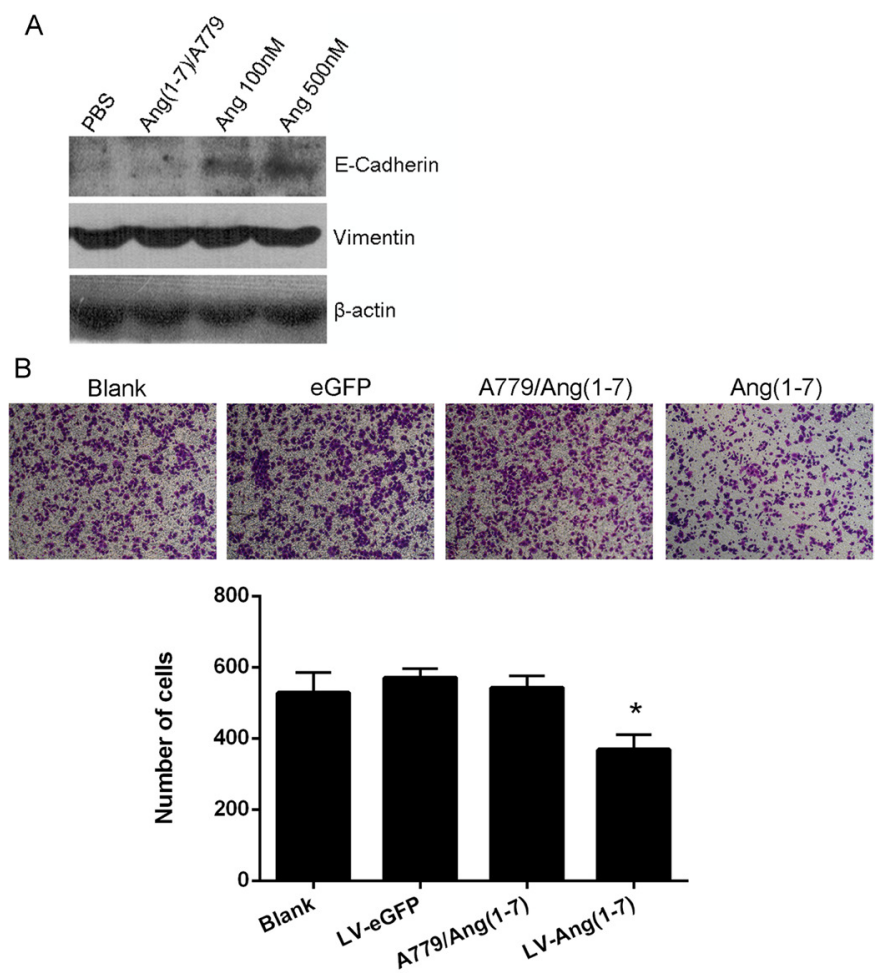

Figure 4: Effects of Ang-(1-7) on Spc-A1 cell EMT. (A) Western blot analysis of E-Cadherin and Vimentin protein expression in Spc-A1 cells after treatment with PBS, $1 \mu \mathrm{M}$ A779 with $500 \mathrm{nM}$ Ang-(1-7), $100 \mathrm{nM}$ or $500 \mathrm{nM}$ Ang-(1-7). Western blots are representative of three different experiments. (B) In vitro transwell migration assay. Non-transduced or Lenti-Ang-(1-7) transduced Spc-A1 cells were resuspended in serum free DMEM with or without $1.0 \mu \mathrm{mol} / \mathrm{L}$ the Ang- (1-7) receptor antagonist, A-779, and placed in the top portion of a transwell chamber. Migration ability of Spc-A1 cell was quantified by counting numbers of cells that adhere to the outside surface of the membranes. Five fields per well were counted. Values are expressed as mean $\pm \mathrm{SD}$ of three independent experiments. ${ }^{*} P<0.05$, vs.controls. 
with antibodies against vascular endothelial growth factor (VEGF), exhibited significantly less stained cells in the Ang-(1-7)-treated group compared with the two control groups, suggesting that Ang-(1-7) reduces Spc-A1 xenograft tumor cell angiogenesis (Figure 8A). Vessels were identified by positive immunoreactivity to CD34 in combination with vessel morphology. A marked reduction in immunoreactive CD34-stained vessels was observed in tumor tissue sections from mice treated with AAV-Ang-(1-7) (3.5 \pm 1.5 vessels/field) when compared with mice treated with AAV-eGFP $(15 \pm 5$ vessels/field $)$ or PBS (13 \pm 3 vessels/field; Figure $8 \mathrm{~B})$, suggesting that Ang-(1-7) significantly attenuates tumor vascularization in xenograft lung tumors.

\section{DISCUSSION}

In this study we investigated the effects of Ang(1-7) on the growth of lung cancer in vitro and in vivo, by using lentiviral or AAV vectors expressing fusion proteins which release the heptapeptide. Ang-(1-7) has been shown to inhibit the growth of lung cancer cells through a MAP
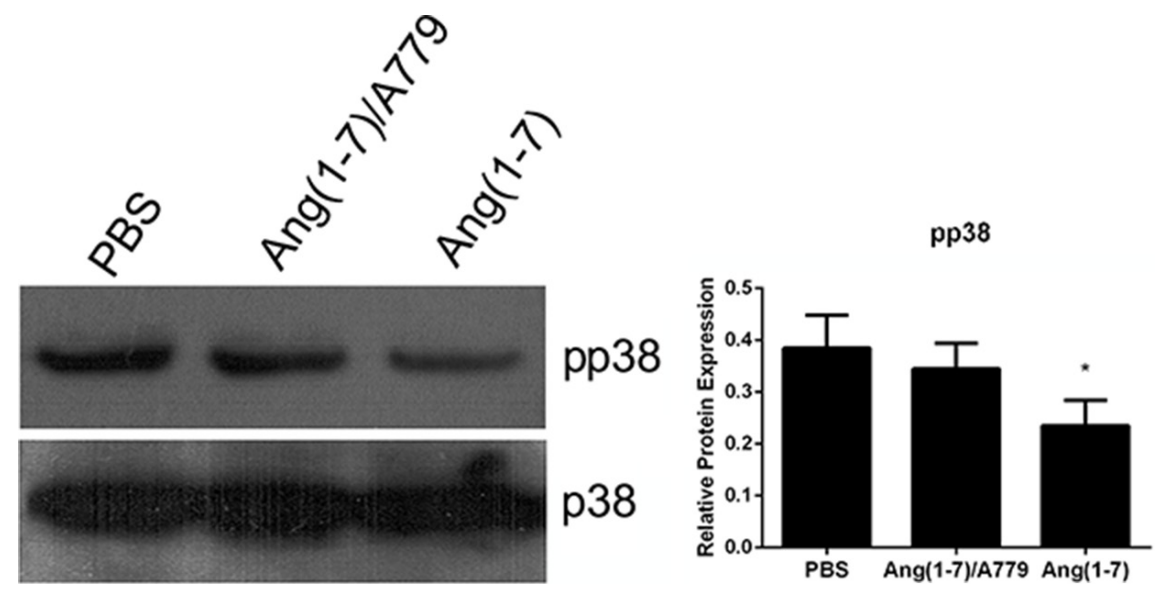

Figure 5: Effects of Ang-(1-7) on p38 MAPK in Spc-A1 cells. Spc-A1 cells were treated with 500 nM Ang-(1-7) with or without $1 \mu \mathrm{M}$ A779. After 3 days of incubation, cells were collected and subjected Western blot analyses of total p38 and pp38. Western blots are representative of three different experiments. $* P<0.01$.
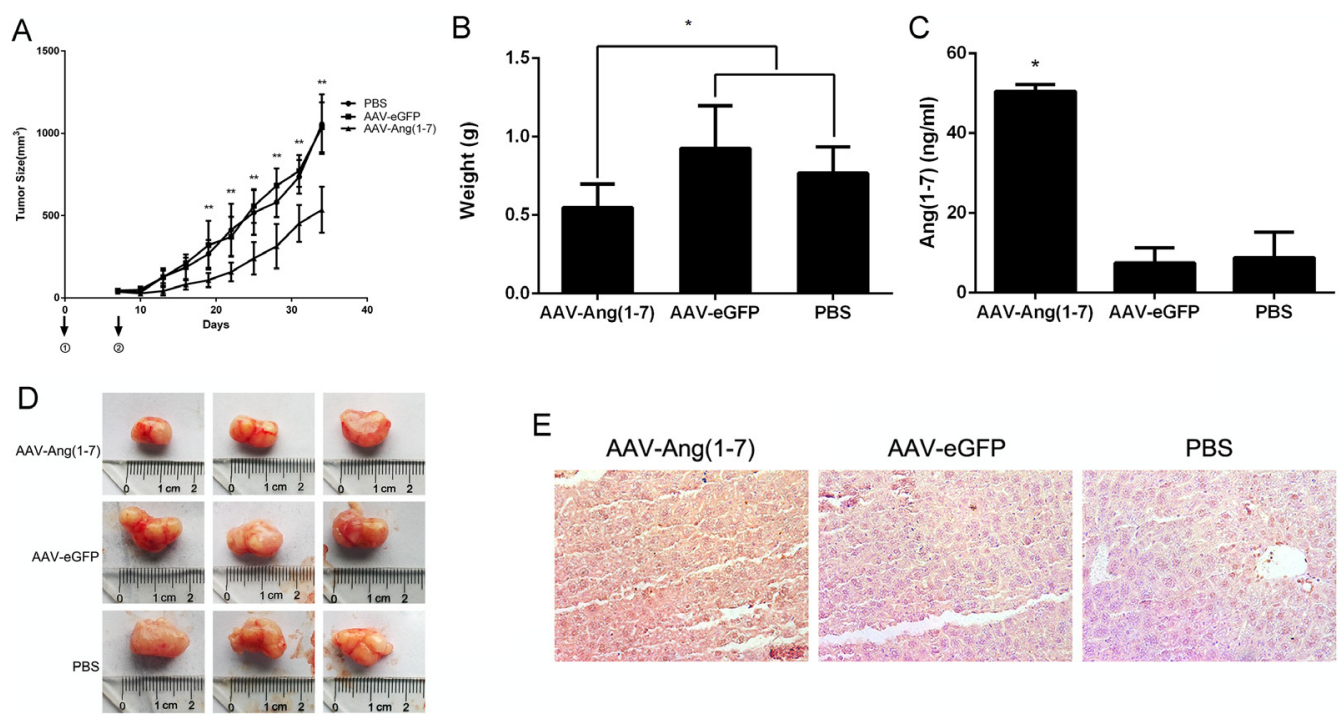

$\mathrm{E}$

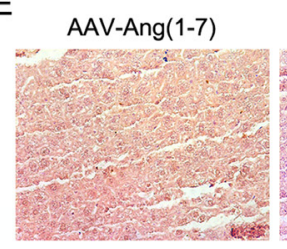

AAV-eGFP

PBS

Figure 6: Ang-(1-7) inhibits lung tumor growth. (A) Growth curve of lung tumor xenografts volumes. Volume $=\left(\mathrm{D} \times \mathrm{d}^{2}\right) / 2$, where $\mathrm{D}$ is the longest diameter and $\mathrm{d}$ is the shortest diameter; $* P<0.01 ; n=6$ (B) Tumors from mice treated with AAV8 (Y733F)-CBAAng-(1-7), AAV8 (Y733F)-CBA-eGFP, or PBS were weighed at the time of sacrifice; ${ }^{*} P<0.01 ; n=6$. Each data point represents the mean \pm SD of 6 mice. $(C)$ Levels of Ang-(1-7) in the sera of controls (AAV-eGFP and PBS-treated mice) and AAV8 (Y733F)-CBA-Ang(1-7)-treated mice, which were determined by ELISA. $* P<0.01$ compared with the control groups. (D) Size of human lung tumor xenografts from mice injected with AAV8 (Y733F)-CBA-Ang-(1-7), AAV8 (Y733F)-CBA-eGFP, or PBS. (E) Sections of livers from treated mice were stained with mouse IgG to indirectly determine Ang-(1-7) overexpression. Representative photomicrographs are shown. 


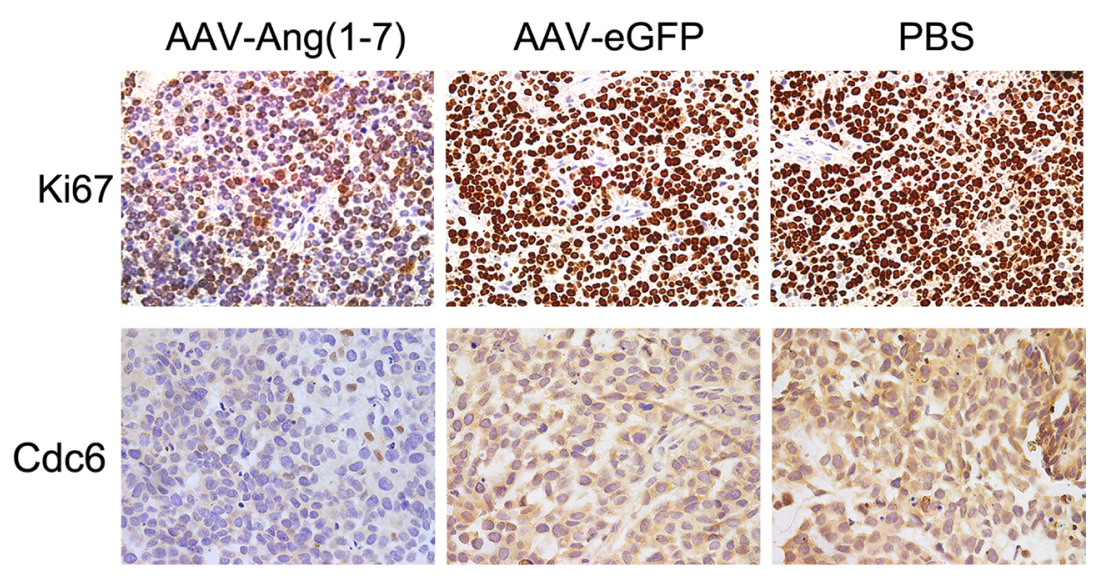

Ki67
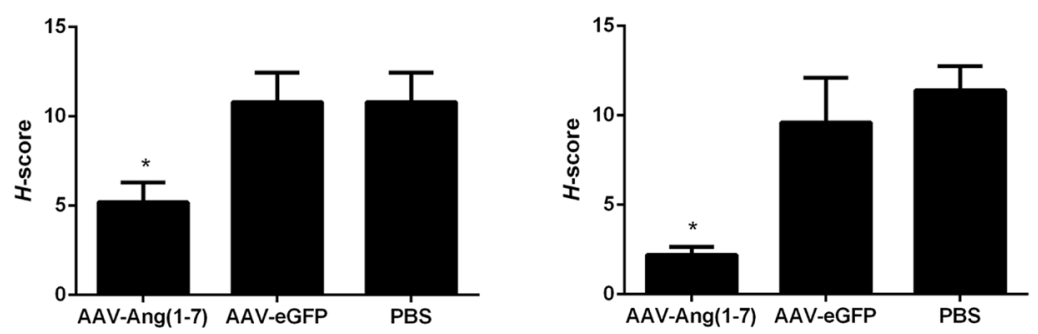

Figure 7: Ang-(1-7) reduces Spc-A1 xenograft tumor cell proliferation. Sections of transplanted tumors infused with AAV8 (Y733F)-CBA-Ang-(1-7), AAV8 (Y733F)-CBA-eGFP, or PBS were stained with Ki67 and Cdc6 antibodies. Representative photomicrographs are shown at the top(magnification, $\times 400)$; The histochemical score $(H$-score) as the final score was achieved by the intensity multiplied by the percentage of positive cells; bars, SD. ${ }^{*} P<0.01$ vs. the control groups (AAV-eGFP and PBS-treated mice).

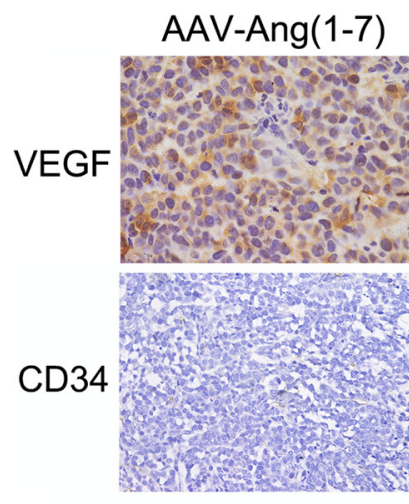

VEGF

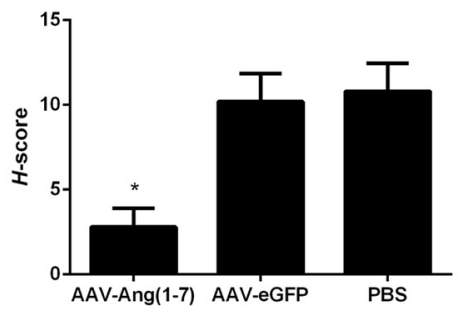

AAV-eGFP
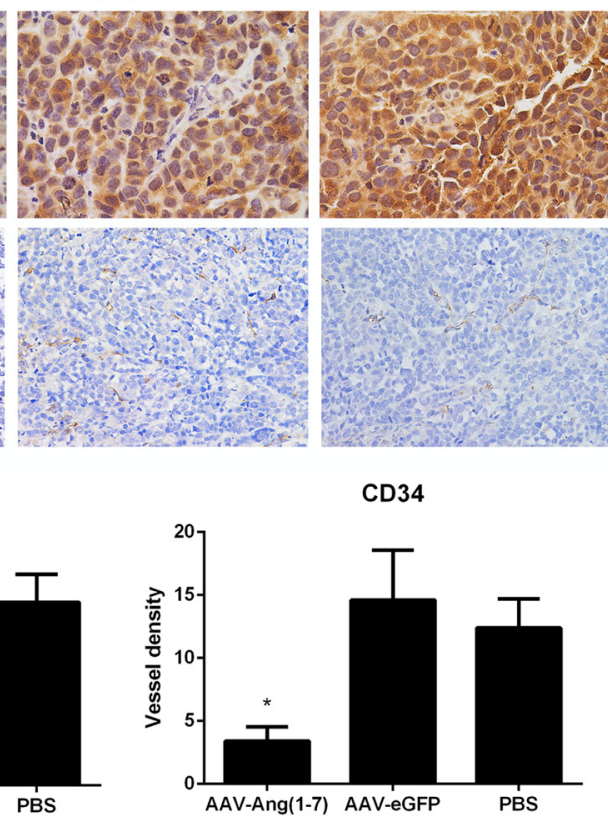

Figure 8: Effect of Ang-(1-7) on angiogenic factors and vessel density in lung tumor xenografts. Ang-(1-7) decreases VEGF expression in human lung cancer xenografts. VEGF expression in tumor sections from AAV8 (Y733F)-CBA-Ang-(1-7), AAV8 (Y733F)CBA-eGFP or PBS-treated human lung cancer xenografts were determined through VEGF immunostaining (magnification $\times 400)$. Blood vessels in tumor sections from lung cancer xenografts were identified by CD34 immunoreactivity and vessel morphology, and quantified as the average of 5 fields selected per tumor (magnification $\times 200$ ). Columns are means from 5 separate experiments; bars, SD. $* P<0.01$ vs. the control groups (AAV-eGFP and PBS-treated mice). 
kinase-dependent mechanism, and attenuated the DNA synthesis in SK-LU-1 cells, effects that were blocked by the Mas receptor antagonist A779 [4]. In the current study, we observed the significant Ang-(1-7)-induced inhibition of the growth of and DNA synthesis in Spc-A1 and A549 (Figures 1 and 2). Cell division cycle 6 (CDC6) is an essential regulator of DNA replication in eukaryotic cells. Its best-characterized function is the assembly of pre-replicative complexes at origins of replication during the G1 phase of the cell division cycle [15]. Prior to DNA replication, pre-replication complexes (pre-RCs) must be assembled at the replication sites in cells in the G1 phase [16]. Cdc6 plays a crucial role in the assembly of pre-RCs by linking the origin recognition complex (Orc) with minichromosome maintenance $(\mathrm{Mcm})$ proteins to form pre-RCs at the sites of DNA replication [17]. Our results revealed that the Ang-(1-7) peptide may inhibit DNA synthesis by decreasing the pre-RCs protein levels, including CDC6 and Mcms (Figure 3).

High levels of CDC6 have been recently reported in around 50\% of NSCLC [12], brain cancer [13] and a subset of mantle cell lymphomas [14], which suggests that CDC6 has oncogenic properties. Our study demonstrated that CDC6 was significantly decreased in lung cancer cells treated with Ang-(1-7), both in vitro or in vivo (Figure 7).

It has been reported that overexpression of CDC6 induced EMT [10], and that activation of p38 triggers EMT and cell migration [18]. Our results indicated that Ang-(1-7) inhibited Spc-A1 cells from undergoing EMT and cell migration (Figure 4), and also inhibited phosphoactivation of p38 MAPK which mediated cell migration [19-22] (Figure 5) was decreased as well. The downregulation of p38 MAPK, ERK1/2, and Akt decreased the protein expression of CDC6 and the activation of the p38 MAPK, and ERK1/2, and Akt pathways are required for the protein stabilization of CDC6 [23]. Based upon this, we suggest that EMT can be a new mechanism in the further research of Ang-(1-7).

Interestingly it has been demonstrated that Ang(1-7) enhanced the migratory and invasive abilities of renal cell carcinoma 786-O and Caki-1 cells in wound-healing, transwell migration and transwell invasion assays [24], effects that are opposite to those in our experiments on lung cancer cell migration (Figure 2). These inconsistent results suggest that the actions of Ang-(1-7) are possibly tumor-specific and highly dependent on its target in different cancer cells [25].

Using mouse models, many studies have shown that Ang-(1-7) exerts effects on tumor growth. Namely, it elicits markedly reduced tumor volume and wet weight of human A549 lung tumors grown in the flank $[5,26]$; of orthotopic human breast tumors positive for the estrogen receptor (BT-474 or ZR-75-1) and overexpressed HER2 (BT-474) in the mammary fat pad [27]; of human LNCaP prostate cancer xenografts [28] as compared to tumors from control animals [3]. All of these studies treated the mice with Ang-(1-7) by inserting minipumps to produce continuous infusion, because of the rapid degradation of the peptide. Our previous study demonstrated that administration of AAV8-(Y733F)-CBAAng-(1-7) significantly attenuated the growth of human nasopharyngeal carcinoma xenograft tumors, suggesting that AAV-based gene therapy is potentially effective and useful for nasopharyngeal cancer treatment [25]. In the present study, we used recombinant adeno-associated virus AAV8-(Y733F)-CBA-Ang-(1-7) to treat nude mice with lung cancer xenografts. The results demonstrated that the growth of Spc-A1 lung cancer cells xenografts were inhibited by AAV8-Ang-(1-7) in vivo (Figure 6A), through the inhibition of cell proliferation and angiogenesis (Figures 7, 8). After a single injection through the tail vein, a high level of Ang-(1-7) was express stably for a month, as detected by ELISA (Figure 6C). Moreover, AAV vectors have been utilized in many clinical trials, which have proven their safety and their therapeutic efficacy in monogenic diseases and also cancers.

In conclusion, our research has demonstrated that Ang-(1-7) inhibited the growth of lung cancer cells, an effect that is probably mediated by the inhibition of DNA synthesis. AAV8-(Y733F)-CBA-Ang-(1-7) significantly attenuated the growth and angiogenesis of lung cancer cells xenograft, suggesting that AAV-based gene therapy can be a useful and efficient approach for treating lung cancer.

\section{MATERIALS AND METHODS}

\section{Cell culture}

Human lung cancer cell lines (A549 and Spc-A1) were obtained from the American Tissue Culture Collection and were cultured in Dulbecco's modified Eagle's medium (DMEM; Corning) supplemented with $10 \% \mathrm{FBS}$ under $5.0 \% \mathrm{CO}_{2}$. Sera and media were purchased from Invitrogen and American Type Culture Collection. HEK 293T cells were cultured in Dulbecco's modified Eagle's medium (DMEM; Corning).

\section{Viral vector construction and preparation}

The lentiviral vector LV-Ang-(1-7) construct used to produce Ang-(1-7) was designed, prepared, and titrated as previously described [7, 29, 30]. Both IgG2b and Ang(1-7) from the fusion protein can be detected intracellularly. The enhanced GFP (eGFP) lentiviral vector LV-eGFP was used as a control. Two adenoassociated viral vectors (AAV), AAV2-chicken $\beta$-actin promoter (CBA)-Ang-(1-7) and AAV2-CBA-eGFP, were constructed as detailed previously [31]. The vector plasmid was packaged in AAV serotype 8 containing a Y733F mutation by transfection of HEK 293T cells according to previously published methods $[32,33]$. Vector doses were expressed as genome copies. 


\section{Quantification of cell proliferation}

For the cell counting assay, cells were infected with LV-Ang-(1-7) to stably overexpress Ang-(1-7). After 72 hours of transduction, the cells were plated in 24 well plates at $1.0 \times 10^{4}$ per well. Cells were harvested on days $1,2,3$, and 4 from triplicate wells and counted using a hemocytometer, to quantify the cell proliferation.

\section{In vitro migration assay}

Cell migration assays were performed using 24-well Transwell plates $(8-\mu \mathrm{m}$ pore size; Corning). Confluent monolayers of Spc-A1 cells transduced with LV-eGFP and LV-Ang-(1-7) with or without A779 were cultured in serum free media in the upper chambers $\left(5 \times 10^{4}\right.$ cells per chamber). The lower chambers were filled with medium DMEM, 10\% FBS. Transwell plates were then incubated at $37^{\circ} \mathrm{C}$ for 24 hours. Cells migrated to the outer side of the upper chamber were fixed with methanol for 30 min, PBS washed three times, and then stained with Wright Giemsa staining. The number of migrated cells was assessed by counting cells from 5 random fields per well. Each experiment was performed in duplicate and repeated three times.

\section{BrdU incorporation assay}

The proliferation of A549 and Spc-A1 cells were determined via 5-bromo-2'deoxyuridine-5'monophosphate (BrdU) incorporation assay. A549 and Spc-A1 cells treated with PBS or $500 \mathrm{nM}$ Ang-(1-7) were seeded into 6-well plates $\left(3 \times 10^{5}\right.$ cells/well $)$ with or without the A-779 $(1.0 \mu \mathrm{mol} / \mathrm{L})$ (Bachem) for $24 \mathrm{~h}$. Two hours prior to fixing the cells, $20 \mu \mathrm{M}$ BrdU (Sigma chemicals) were added to the cultures. Next, the cells were permeabilized with $0.25 \%$ Triton X-100 and $3 \mathrm{M}$ hydrochloric acid. The cells were blocked in goat serum albumin. Then, immunofluorescence was performed as previously described [34] by incubation with an anti-BrdU antibody (1:1000, Cell Signaling Technology, USA) at $4^{\circ} \mathrm{C}$ overnight. The cells were then washed with cold PBS and incubated with Goat anti-Mouse IgG $(\mathrm{H}+\mathrm{L})$ Secondary Antibody, Cy3 conjugate (Invitrogen, USA) for $1 \mathrm{~h}$ at room temperature. Labeling indices were calculated as the number of positively stained cells divided by the number of total cells.

\section{RNA isolation, reverse transcription, and quantitative real-time RT-PCR}

Total RNA was extracted using a HiPure Total RNA Kit (Magen) according to the manufacturer's instructions. Quantitative realtime RT-PCR was performed on an ABI 7500 real-time PCR system (Applied Biosystems) as described previously [35]. The samples were quantified by the comparative $\Delta \Delta \mathrm{Ct}$ method by using human GAPDH as the internal standard.

\section{Western blotting}

Cell lysis and western blotting were performed as described previously [36]. Immunoreactive bands were visualized by enhanced chemiluminescence detection reagents and analyzed with Image $\mathrm{J}$ 1.62. For cell signaling study, $2 \times 10^{5}$ cells were seeded into 6 -well plates and allowed to settle for $24 \mathrm{~h}$. Then the cells were starved in serum-free medium for $24 \mathrm{~h}$ before stimulation with Ang-(1-7) or Ang-(1-7)/A779.

\section{Tumor growth assay}

Female BALB/c nude mice aged 4 to 5 weeks were purchased from the Institute of Comparative Medicine and Center of Laboratory Animals of the Southern Medical University (SMU). Animal handling and experimental procedures were approved by the Animal Experimental Ethics Committee of SMU. Athymic mice were subjected to s.c. injections of human Spc-A1 lung cancer cells $\left(1.0 \times 10^{6}\right)$ in Matrigel (50:50) into the lower flank to induce tumor growth. After the tumors reached $\sim 50 \mathrm{~mm}^{3}$, the mice were placed into three groups at random and the animals received tail vein injections of AAV8 (Y733F)-CBA-Ang-(1-7), AAV8 (Y733F)-CBAeGFP $\left(1 \times 10^{11} \mathrm{vg} /\right.$ mice $)$ or PBS. Each group contained 5 mice and the experiment was repeated 3 times. Tumor size was measured every 3 days. The mice were anesthetized on day 32 and euthanized by decapitation and tumors were dissected. Tumor volumes were calculated as follows: volume $=\left(\mathrm{D} \times \mathrm{d}^{2}\right) / 2$, where $\mathrm{D}$ meant the longest diameter and $\mathrm{d}$ meant the shortest diameter. Both livers and tumors were fixed in $10 \%$ buffered formalin and used for histologic and immunohistochemical analysis. Sera were also collected for quantification of Ang-(1-7) by ELISA (Bachem).

\section{Immunohistochemistry}

Tumors were fixed in formalin for 24 hours and incubated in $70 \%$ ethanol for 48 hours before embedding in paraffin. The embedded tumors were cut into 5-mmthick sections and stained with $\mathrm{H} \& \mathrm{E}$ to determine morphology. Cell proliferation in the transplanted tumors was analyzed using antibodies to Ki67 (1:200; Abcam) and Cdc6 (1:300; Abcam) expression. Angiogenesis was determined by immunostaining with antibodies to VEGF (1:100; Wanleibio, China) and CD34 (1:200; Abcam). Visualization was achieved using the EnVision+ peroxidase system (Dako) according to the manufacturer's protocols. Ki67- immunoreactive cells were expressed as a percentage of the total cell number of examined fields. Blood vessels were visualized by the presence of 
CD34-immunostained endothelial cells and identified by their morphology, as vessels cut in cross-section with visible lumens or vessels cut longitudinally with tubelike morphology [37]. The vessel density was assessed by counting vessels from five random fields per tumor. Counts were done by an individual who was blinded as to the treatment.

\section{Evaluation of immunostaining scores}

The intensity of staining was scored by applying a semiquantitative system, ranging from negative to strong as follows: 0 (negative), 1 (weak), 2 (moderate), and 3 (strong). The percentage of positive cells was categorized according to the positive tumor cells: $<25 \%$ is $1 ; 25 \%$ to $50 \%$ is $2 ; 51 \%$ to $75 \%$ is 3 ; and $>75 \%$ is 4 . The histochemical score ( $H$-score) as the final score was achieved by the intensity multiplied by the percentage of positive cells $[38,39]$.

\section{ACKNOWLEDGMENTS}

We acknowledge Guoguo Yi, Yue He for their help with animal experiments.

\section{CONFLICTS OF INTEREST}

The authors have declared that no conflicts of interests exist.

\section{GRANT SUPPORT}

This work was supported by National Natural Science Foundation of China Grant 81072113 (H. Li), National 863 High Technique Development Project of China Grant 2012AA02A403 (H. Li), and in part from National Natural Science Foundation of China Grants 81272482 (J.Li), 81401920 (H.Du) as well as by awards the Doctoral Program of Higher Education of China Grant 20134433120020 (H. Du).

\section{REFERENCES}

1. Song X, Xie L, Wang X, Zeng Q, Chen TC, Wang W, Song X. Temozolomide-perillyl alcohol conjugate induced reactive oxygen species accumulation contributes to its cytotoxicity against non-small cell lung cancer. Sci Rep. 2016; 6:22762.

2. Evans M. Lung cancer: needs assessment, treatment and therapies. Br J Nurs. 2013; 22:S15-S16, S18, S20-S22.

3. Gallagher PE, Arter AL, Deng G, Tallant EA. Angiotensin-(1-7): a peptide hormone with anti-cancer activity. Curr Med Chem. 2014; 21:2417-2423.
4. Gallagher PE, Tallant EA. Inhibition of human lung cancer cell growth by angiotensin-(1-7). Carcinogenesis. 2004; 25:2045-2052.

5. Menon J, Soto-Pantoja DR, Callahan MF, Cline JM, Ferrario CM, Tallant EA, Gallagher PE. Angiotensin-(1-7) Inhibits Growth of Human Lung Adenocarcinoma Xenografts in Nude Mice through a Reduction in Cyclooxygenase-2. Cancer Res. 2007; 67:2809-2815.

6. Rodgers KE, Oliver J, DiZerega GS. Phase I/II dose escalation study of angiotensin 1-7 [A(1-7)] administeredbefore and after chemotherapy in patients with newly diagnosed breastcancer. Cancer Chemoth Pharm. 2006; 57:559-568.

7. Qi Y, Shenoy V, Wong F, Li H, Afzal A, Mocco J, Sumners C, Raizada MK, Katovich MJ. Lentivirus-mediated overexpression of angiotensin-(1-7) attenuated ischaemiainduced cardiac pathophysiology. Exp Physiol. 2011; 96:863-874.

8. Ferrario CM, Chappell MC, Tallant EA, Brosnihan KB, Diz DI. Counterregulatory actions of angiotensin-(1-7). Hypertension. 1997; 30:535-541.

9. Tallant EA, Ferrario CM, Gallagher PE. Angiotensin-(1-7) inhibits growth of cardiac myocytes through activation of the mas receptor. Am J Physiol Heart Circ Physiol. 2005; 289:H1560-H1566.

10. Chen CH, Lin DS, Cheng CW, Lin CJ, Lo YK, Yen CC, Lee AY, Hsiao CD. Cdc6 cooperates with c-Myc to promote genome instability and epithelial to mesenchymal transition EMT in zebrafish. Oncotarget. 2014; 5:6300-6311. doi: 10.18632/oncotarget.2204.

11. Wheelock MJ, Johnson KR. Cadherins as modulators of cellular phenotype. Annu Rev Cell Dev Biol. 2003; 19: 207-235.

12. Karakaidos $\mathrm{P}$, Taraviras $\mathrm{S}$, Vassiliou LV, Zacharatos $\mathrm{P}$, Kastrinakis NG, Kougiou D, Kouloukoussa M, Nishitani H, Papavassiliou AG, Lygerou Z, Gorgoulis VG. Overexpression of the replication licensing regulators hCdt 1 and hCdc 6 characterizes a subset of non-small-cell lung carcinomas: synergistic effect with mutant p53 on tumor growth and chromosomal instability - evidence of E2F-1 transcriptional control over hCdt1. Am J Pathol. 2004; 165:1351-1365.

13. Ohta S, Koide M, Tokuyama T, Yokota N, Nishizawa S, Namba H. Cdc6 expression as a marker of proliferative activity in brain tumors. Oncol Rep. 2001; 8:1063-1066.

14. Pinyol M, Salaverria I, Bea S, Fernandez V, Colomo L, Campo E, Jares P. Unbalanced expression of licensing DNA replication factors occurs in a subset of mantle cell lymphomas with genomic instability. Int J Cancer. 2006; 119:2768-2774.

15. Borlado LR, Mendez J. CDC6: from DNA replication to cell cycle checkpoints and oncogenesis. Carcinogenesis. 2008; 29:237-243. 
16. Stillman B. Cell cycle control of DNA replication. Science. 1996; 274:1659-1664.

17. Saxena S, Dutta A. Geminin-Cdt1 balance is critical for genetic stability. Mutat Res. 2005; 569:111-121.

18. Cho JH, Hong WG, Jung YJ, Lee J, Lee E, Hwang SG, Um HD, Park JK. Gamma-Ionizing radiation-induced activation of the EGFR-p38/ERK-STAT3/CREB-1-EMT pathway promotes the migration/invasion of non-small cell lung cancer cells and is inhibited by podophyllotoxin acetate. Tumour Biol. 2016; 37:7315-7325.

19. Yu J, Bian D, Mahanivong C, Cheng RK, Zhou W, Huang S. p38 Mitogen-activated protein kinase regulation of endothelial cell migration depends on urokinase plasminogen activator expression. J Biol Chem. 2004; 279:50446-50454.

20. Yang Y, Cheon S, Jung MK, Song SB, Kim D, Kim HJ, Park H, Bang SI, Cho D. Interleukin-18 enhances breast cancer cell migration via down-regulation of claudin-12 and induction of the p38 MAPK pathway. Biochem Biophys Res Commun. 2015; 459:379-386.

21. Shen YJ, Zhu XX, Yang X, Jin B, Lu JJ, Ding B, Ding ZS, Chen SH. Cardamonin inhibits angiotensin IIinduced vascular smooth muscle cell proliferation and migration by downregulating p38 MAPK, Akt, and ERK phosphorylation. J Nat Med. 2014; 68:623-629.

22. Lee HM, Lee CK, Lee SH, Roh HY, Bae YM, Lee KY, Lim J, Park PJ, Park TK, Lee YL, Won KJ, Kim B. p38 mitogen-activated protein kinase contributes to angiotensin II-stimulated migration of rat aortic smooth muscle cells. J Pharmacol Sci. 2007; 105:74-81.

23. Y L, Hock JM, Sullivan C, G F, Cox AJ, Davis KT, Davis BH, X L. Activation of the p38 MAPK/Akt/ERK1/2 Signal Pathways Is Required for the Protein Stabilization of CDC6 andCyclin D1 in Low-Dose Arsenite-Induced Cell Proliferation. J Cell Biochem. 2010; 111:1546-1555.

24. Zheng S, Yang Y, Song R, Yang X, Liu H, Ma Q, Yang L, Meng R, Tao T, Wang S, He J. Ang-(1-7) promotes the migration and invasion of human renal cell carcinoma cells via Mas-mediated AKT signaling pathway. Biochem Biophys Res Commun. 2015; 460:333-340.

25. Pei N, Wan R, Chen X, Li A, Zhang Y, Li J, Du H, Chen B, Wei W, Qi Y, Zhang Y, Katovich MJ, Sumners C, et al. Angiotensin-(1-7) Decreases Cell Growth and Angiogenesis of Human Nasopharyngeal Carcinoma Xenografts. Mol Cancer Ther. 2016; 15:37-47.

26. Soto-Pantoja DR, Menon J, Gallagher PE, Tallant EA. Angiotensin-(1-7) inhibits tumor angiogenesis in human lung cancer xenografts with a reduction in vascular endothelial growth factor. Mol Cancer Ther. 2009; 8:1676-1683.

27. Cook KL, Metheny-Barlow LJ, Tallant EA, Gallagher PE. Angiotensin-(1-7) Reduces Fibrosis in Orthotopic Breast Tumors. Cancer Res. 2010; 70:8319-8328.

28. Krishnan B, Smith TL, Dubey P, Zapadka ME, Torti FM, Willingham MC, Tallant EA, Gallagher PE.
Angiotensin-(1-7) Attenuates Metastatic Prostate Cancer and Reduces Osteoclastogenesis. The Prostate. 2013; 73:71-82.

29. Methot D, VanKats JP, Lochard N, Tremblay F, Silversides DW, Reudelhuber TL. Development and application of a biological peptide pump for the study of the in vivo actions of angiotensin peptides. Am J Hypertens. 2001; 14:38S-43S.

30. Ferreira AJ, Castro $\mathrm{CH}$, Guatimosim S, Almeida PW, Gomes ER, Dias-Peixoto MF, Alves MN, FagundesMoura CR, Rentzsch B, Gava E, Almeida AP, Guimaraes AM, Kitten GT, et al. Attenuation of isoproterenol-induced cardiac fibrosis in transgenic rats harboring an angiotensin-(1-7)producing fusion protein in the heart. Ther Adv Cardiovasc Dis. 2010; 4:83-96.

31. Li H, Gao Y, Qi Y, Katovich MJ, Jiang N, Braseth LN, Scheuer DA, Shi P, Sumners C. Macrophage migration inhibitory factor in hypothalamic paraventricular nucleus neurons decreases blood pressure in spontaneously hypertensive rats. Faseb J. 2008; 22:3175-3185.

32. Petrs-Silva H, Dinculescu A, Li Q, Min SH, Chiodo V, Pang JJ, Zhong L, Zolotukhin S, Srivastava A, Lewin AS, Hauswirth WW. High-efficiency transduction of the mouse retina by tyrosine-mutant AAV serotype vectors. Mol Ther. 2009; 17:463-471.

33. Zolotukhin S, Byrne BJ, Mason E, Zolotukhin I, Potter M, Chesnut K, Summerford C, Samulski RJ, Muzyczka N. Recombinant adeno-associated virus purification using novel methods improves infectious titer and yield. Gene Ther. 1999; 6:973-985.

34. Chen S, Wan P, Ding W, Li F, He C, Chen P, Li H, Hu Z, Tan W, Li J. Norcantharidin inhibits DNA replication and induces mitotic catastrophe by degrading initiation protein Cdc6. Int J Mol Med. 2013; 32:43-50.

35. Pei N, Jie F, Luo J, Wan R, Zhang Y, Chen X, Liang Z, Du H, Li A, Chen B, Zhang Y, Sumners C, Li J, et al. Gene expression profiling associated with angiotensin II type 2 receptor-induced apoptosis in human prostate cancer cells. Plos One. 2014; 9:e92253.

36. Marcus JS, Karackattu SL, Fleegal MA, Sumners C. Cytokine-stimulated inducible nitric oxide synthase expression in astroglia: role of Erk mitogen-activated protein kinase and NF-kappaB. Glia. 2003; 41:152-160.

37. Weidner N. Current pathologic methods for measuring intratumoral microvessel density within breast carcinoma and other solid tumors. Breast Cancer Res Treat. 1995; 36:169-180.

38. Friedrichs K, Gluba S, Eidtmann H, Jonat W. Overexpression of p53 and prognosis in breast cancer. Cancer-Am Cancer Soc. 1993; 72:3641-3647.

39. McCarty KJ, Miller LS, Cox EB, Konrath J, McCarty KS. Estrogen receptor analyses. Correlation of biochemical and immunohistochemical methods using monoclonal antireceptor antibodies. Arch Pathol Lab Med. 1985; 109:716-721. 